Published online 2017 April 13.

Abstract

\title{
What Should We Know Before Doing Mechanical Thrombectomy in Stroke Patients
}

\author{
Ramin Pourghorban ${ }^{1, *}$ \\ ${ }^{1}$ Department of Radiology, Imam Khomeini Hospital Complex, Tehran University of Medical Sciences, Tehran, Iran \\ "Corresponding author: Ramin Pourghorban, MD, Department of Radiology, Imam Khomeini Hospital Complex, Tehran University of Medical Sciences, Tehran, Iran. E-mail: \\ ramin_p2005@yahoo.com
}

Received 2016 December 21; Accepted 2017 February 08.

\begin{abstract}
Mechanical thrombectomy has shown promising results in large vessel ischemic stroke, with recent large trials changing to guidelines worldwide. In this presentation, results from the five recent randomized trials will be discussed. There is an ongoing debate on the inclusion and exclusion criteria of mechanical thrombectomy. It can be done in large vessel occlusions, including up to M2 middle cerebral artery (MCA), anterior cerebral artery (ACA), basilar artery, and posterior cerebral artery (PCA). The effect of time window, age, prior intravenous thromblysis on patients' selection will be discussed. An elaboration on the effect of national institutes of health stroke scale (NIHSS) score in the clinical setting, Alberta stroke program early computed tomography score (ASPECTS) on non-contrast CT, presence of collaterals on CT angiography (CTA), and presence of penumbra on CT perfusion or post contrast MRI will be given as well. The pros and cons of general anesthesia versus conscious sedation during mechanical thrombectomy and aspiration technique versus stent-retriever will be reviewed. The usual devices used in mechanical thrombectomy and practical tips of endovascular treatment will be discussed. Finally, modified treatment in cerebral ischemia (mTICI) score after thrombectomy will be shown.
\end{abstract}

This is an abstract presented in the 33rd Iranian congress of radiology (ICR) and the 15th congress of Iranian radiographic science association (IRSA). 